\title{
Balloon expandable stents for systemic venous pathway stenosis late after Mustard's operation
}

\author{
F A Bu'Lock, A J P Tometzki, D J Kitchiner, R Arnold, I Peart, K P Walsh
}

\begin{abstract}
Objectives-Description and evaluation of current experience with the use of balloon expandable stents for the relief of systemic venous pathway stenosis late after Mustard's operation.

Design-Retrospective observational study of technical procedures, angiographic, and haemodynamic findings.

Patients-Twenty long term survivors of Mustard's operation for transposition of the great arteries (TGA) with angiographic evidence of systemic venous pathway narrowing.

Intervention-Systemic venous pathway stenoses were stented using balloon expandable Palmaz stents.

Results-Twenty seven stents were deployed across 24 stenoses. Seventeen stents were placed in the inferior baffle (16 patients), with an increase in mean (range) minimum diameter from 9.6 (4.5$15.9)$ to $16.5(11.9-22.2) \mathrm{mm}(p=0.007)$, and a reduction in mean pressure gradient from $3.1(0-8)$ to $0.67(0-3)$ mm Hg $(p=0.002)$. Eight stents were placed in the superior pathways of eight patients, with diameters widened from 9.1 $(3.5-14.1)$ to $15.2 \quad(8.7-19.2) \mathrm{mm}$ $(p=0.018)$, and gradients reduced from $6.4(2-11)$ to $0.9(0-2) \mathrm{mm} \mathrm{Hg}(\mathrm{p}=0.02)$. Two badly deployed stents were safely withdrawn from their intracardiac positions and redeployed in the iliac vein. Transvenous pacemaker insertion was facilitated by prior stent insertion.

Conclusions-The use of balloon expandable stents for late systemic pathway narrowing after Mustard's operation is safe and effective. The beneficial effects of stenting are likely to be more durable than those of balloon angioplasty alone, but longer term follow up is required. (Heart 1998;79:225-229)
\end{abstract}

Heart Clinic, Royal Liverpool Children's Hospital, Alder Hey, Eaton Road, Liverpool L12 2AP, UK

F A Bu'Lock

A J P Tometzki

D J Kitchiner

R Arnold

I Peart

K P Walsh

Correspondence to: Dr Bu'Lock.

Accepted for publication 3 October 1997 protein losing enteropathy, peripheral oedema, and ascites. ${ }^{5-7}$ There may also be an increased risk of sudden death, probably related to impaired tolerance of atrial tachyarrhyth- mias. ${ }^{8}{ }^{9}$ Reoperation for baffle obstruction is associated with significant morbidity and mortality, ${ }^{510}$ and baffle stenosis can recur. Balloon angioplasty may be effective in the short term $^{11}{ }^{12}$ but there is a high incidence of restenosis; up to $40 \%$ in our experience. ${ }^{12}$ Endovascular stent implantation for systemic venous pathway stenosis after the Mustard operation has recently been described in a few cases. ${ }^{13-15}$ Our increasing experience in the use of balloon expandable stents for other lesions and our concerns about the long term implications of baffle stenosis (and restenosis after balloon angioplasty) led us to examine their use for this challenging problem in more detail.

\section{Patients and methods}

Given the difficulties with precordial echocardiographic diagnosis of systemic venous baffle narrowing in older patients after Mustard's procedure, ${ }^{16}$ it has been our recent policy to offer angiographic examination of all such patients when they attend for outpatient review. Particular indications for catheterisation would be previous echocardiographic or angiographic evidence of baffle narrowing (regardless of whether previously dilated), as well as patients with impaired effort tolerance, right ventricular dysfunction, or lack of cardiac catheterisation since surgical repair.

Of 154 long term survivors of Mustard-type repair of TGA, 45 underwent cardiac catheterisation between January 1993 and May 1996. Significant angiographic narrowing of superior, inferior or both limbs of the systemic venous pathways (minimum diameter less than two thirds of relevant caval diameter) were identified in 20 patients and treated by stent implantation. There were 15 male and five female patients aged between six and 23 years (mean 15.5), weighing between 21 and $80 \mathrm{~kg}$ (mean 54). Ten patients had undergone balloon angioplasty for baffle stenosis 1.5-7.9 years (mean 4.5) previously but none had undergone late surgical revision. One patient had recently been successfully resuscitated from ventricular fibrillation and one had radiofrequency ablation of atrial flutter immediately before stent implantation. Four patients had mild angiographic narrowings noted on previous catheterisation but had not been treated, and six patients had not undergone catheterisation since surgery. Three of these had possible Doppler echocardiographic evidence of baffle narrowing. The mean time from stenting to last follow up was 11 months (range 3-29). 
TECHNIQUE

Cardiac catheterisation was performed under local $(n=5)$ or general $(n=16)$ anaesthesia, via a percutaneous femoral and/or internal jugular $(n=2)$ venous approach, with additional femoral arterial access for monitoring. Heparin was administered at a dose of 50-100 iu/kg (maximum dose $5000 \mathrm{iu}$ ), and all patients received a single intravenous dose of $30 \mathrm{mg} / \mathrm{kg}$ of cefotaxime (maximum dose $1 \mathrm{~g}$ ). A full systemic venous haemodynamic and oximetric study was performed. Withdrawal pressure gradients across both limbs of the baffle were recorded, any intracardiac shunting was noted and cineangiography was then performed. Superior and inferior caval injections were recorded from both antero-posterior and lateral projections. Stenting of a pathway was undertaken if its minimum diameter was less than two thirds of the juxtacardiac diameter of the appropriate caval vein. Formal measurement of all hard copy angiograms was performed separately at a later date by a single operator.

Following angiography, a $0.035 "$ or 0.038 " superstiff Amplatz (Boston Scientific, UK) wire was placed across the stenosis (or stenoses). The soft tip of the wire was placed in the left ventricle $(n=10)$ or SVC $(n=5)$ for inferior baffle stenoses, and high in the SVC for superior limb obstruction. When the internal jugular approach was used for SVC stenting alone, the wire tip was placed in the left ventricle but for bicaval stenting it was placed in the inferior vena cava (IVC). When stents were to be placed in both systemic pathways, the most distal lesion was treated first. In four patients, the stenosis was predilatated with a balloon of the chosen diameter, but in general stenting was performed as the primary intervention. A long recurved 9-12 F Mullins sheath with haemostatic valve (William Cook, UK) was then advanced over the wire and across the stenosis. The dilator was then removed and the sheath de-aired and flushed.

Balloon catheter selection was based on the estimated diameter of the non-stenotic part of the venous pathways, with a balloon diameter sufficient to ensure full deployment of the stent with complete relief of the stenosis. Some allowance for growth was made in the younger patients. Either Balt Crystal (Merck, UK) $(\mathrm{n}=20)$, or Mansfield (Boston Scientific) $(n=2)$ balloon catheters were used. The balloon was preinflated with dilute (1/4) contrast medium, and deflated. The chosen Johnson and Johnson stent (Cordis, UK) was then mounted on the centre of the balloon and hand crimped into position. A small amount of undiluted contrast medium was applied to improve adherence of the stent to the balloon and facilitate passage through the haemostatic valve into the sheath. During passage of the stent-balloon combination over the guidewire to the site of the stenosis, the position of the stent relative to the radio-opaque balloon markers was carefully monitored by fluoroscopy. The sheath was then withdrawn several centimetres back from the proximal balloon marker and the stent-balloon siting optimised relative to the stenosis using hand or power injections of contrast through the side-arm of the sheath. Balloon inflation for stent deployment was performed under fluoroscopic control. After deflation, the balloon was carefully withdrawn through the deployed stent, with manipulation of the guidewire to keep the deflated balloon central within the stent and minimise the chances of accidental stent displacement. The balloon catheter was then withdrawn through or with the sheath, leaving the guidewire positioned across the stent to facilitate the introduction of an angiographic catheter. Angiography was repeated and a withdrawal gradient obtained in the appropriate order for the stent site. If bicaval stenting was required, the guidewire was repositioned using an end-hole catheter, with particular care to avoid trapping the wire in the mesh of the first stent. A second stent was then deployed in a similar manner to the first.

All patients were monitored in hospital overnight. A chest radiograph and echocardiogram were obtained the following day to document maintenance of stent position and absence of other complications. Patients were discharged on aspirin $75 \mathrm{mg}$ daily for three to six months in addition to any usual medication, with first follow up appointment one month after stent implantation.

\section{STATISTICAL ANALYSIS}

Haemodynamic and angiographic data were examined using standard non-parametric statistical methods. Paired data (pre- and postintervention) were compared using Wilcoxon's signed ranks tests. All p values quoted are twotailed.

\section{Results}

Twenty seven Johnson and Johnson stents (25 P308s, one P188, and one P5015) were deployed across 24 stenoses, during 21 procedures in 20 patients. Seventeen were placed in the inferior limb (16 patients) and eight in the superior limb (eight patients) of the systemic venous pathway.

Table 1 Haemodynamic and angiographic data

\begin{tabular}{|c|c|c|c|c|c|c|c|c|}
\hline & \multicolumn{4}{|c|}{ Dimension (mm) } & \multicolumn{4}{|c|}{ Pressure $(m m \mathrm{Hg})$} \\
\hline & Pre & Post & Change (\%) & $p$ value & Pre & Post & Change (\%) & $p$ value \\
\hline \multicolumn{9}{|l|}{$S V C(n=8)$} \\
\hline Mean (SD) & $9.1(3.5)$ & $15.2(3.8)$ & $80(43)$ & 0.01 & $6.4(3.9)$ & $0.9(0.7)$ & $83(16)$ & 0.018 \\
\hline Range & $3.5-14.1$ & $8.7-19.2$ & $31-149$ & & $2-11$ & $0-2$ & $67-100$ & \\
\hline \multicolumn{9}{|l|}{$\begin{array}{l}\text { IVC }(n=17) \\
\text { Mean (SD) }\end{array}$} \\
\hline Mean (SD) & $9.6(2.8)$ & $16.5(3.3)$ & $83(56)$ & 0.0007 & $3.1(1.8)$ & $0.67(0.9)$ & $76(31)$ & 0.002 \\
\hline Range & $4.5-15.9$ & $11.9-22.2$ & $20-233$ & & $0-8$ & $0-3$ & $0-100$ & \\
\hline
\end{tabular}


Pressure gradients were reduced from a mean of 6.4 to $0.9 \mathrm{~mm} \mathrm{Hg}(\mathrm{p}=0.018)$ in the superior limb and from 3.1 to $0.67 \mathrm{~mm} \mathrm{Hg}$ in the inferior limb $(\mathrm{p}=0.002)$ (table 1). However, severity of stenosis was most apparent from the angiogram, and was clearest and easiest to measure from the antero-posterior projection. Minimum pathway diameter was increased from 9.1 to $15.2 \mathrm{~mm}(\mathrm{p}=0.01)$ in the superior limb and from 9.6 to $16.5 \mathrm{~mm}$ $(p=0.0007)$ in the inferior limb. The ratio of the minimum pathway to caval diameter before stenting was increased from a mean of 0.42 $(0.14-0.63)$ to $0.73(0.45-1.2)(p=0.001)$ for inferior pathways, and $0.44(0.23-0.65)$ to $0.75(0.58-0.94)$ for superior pathways $(\mathrm{p}=0.018)$.

Although not specifically questioned, many patients reported a subjective improvement in effort tolerance following the procedure but the significance of this is difficult to assess. Of the 10 patients with impaired right ventricular function, five were already on angiotensin converting enzyme (ACE) inhibitors and the remainder were started on treatment during the admission for catheterisation; one also
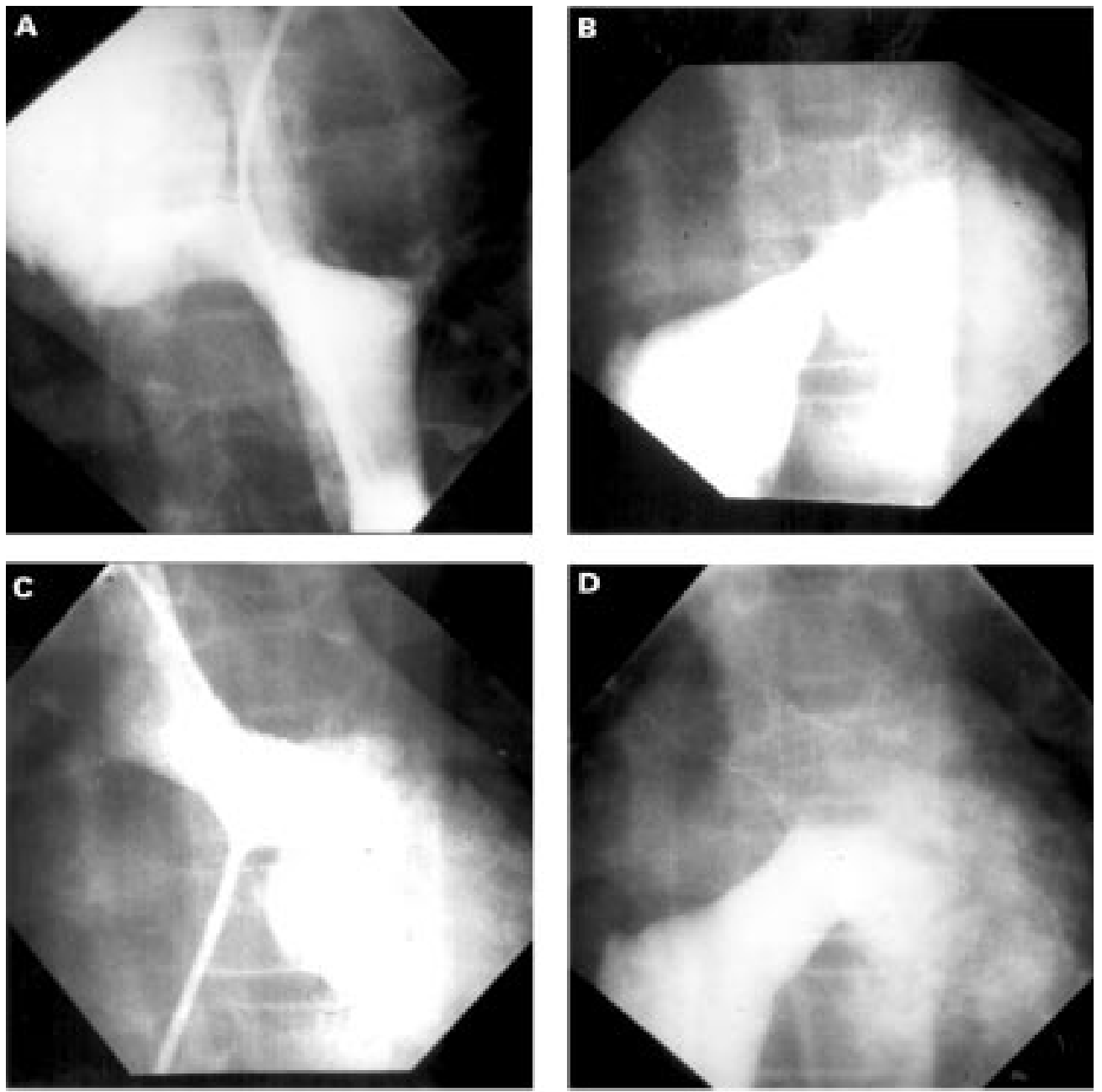

Figure 1 (A) Cineangiogram (antero-posterior projection) demonstrating significant stenosis of superior caval pathway with additional dilatation of the azygos vein. (B) Cineangiogram (antero-posterior projection) demonstrating severe inferior caval pathway obstruction in the same patient as $(A)$. (C) Cineangiogram in same view as $(A)$, after deployment of a P308 stent on a $20 \mathrm{~mm}$ balloon in superior caval narrowing. The azygous vein is no longer readily seen.

(D) Cineangiogram in same view as (B) after deployment of a P308 stent on a $20 \mathrm{~mm}$ balloon in inferior caval stenosis. The superior caval stent is also seen in position. underwent radiofrequency ablation of his arrhythmia. In addition, in four patients, right ventricular failure has progressed; two have died (one post-transplant) and two will require heart transplantation in the near future.

\section{TECHNICAL CONSIDERATIONS}

venous access may be difficult or in these older patients. In two patients with the guidewire positioned in the left ventricle for the patient with superior pathway stenosis and the IVC for bicaval stenting in the other. In a patient with iliac vein occlusion and a long segment IVC narrowing, a hydrophilic guidewire (Kimal; Terumo, UK) was passed to the IVC via collaterals and then exchanged for the superstiff wire.

Most pathway stenoses were very discrete (fig 1), but some were too long for the standard P308 (30 mm long at $8 \mathrm{~mm}$ diameter) stent. In one patient, with a residual waist distal to the initial P308 stent, a shorter P188 stent was added, interdigitating with the first stent to good effect. In a subsequent similar patient, a longer

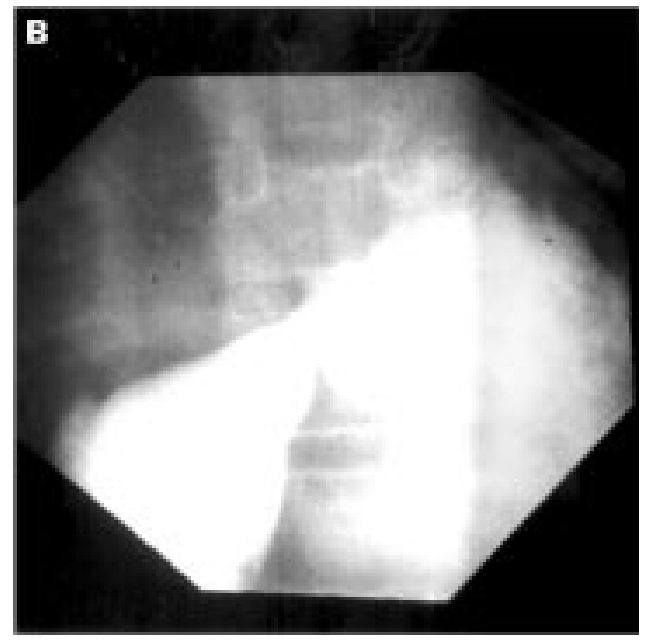


stent (P5015, $50 \mathrm{~mm}$ long at $15 \mathrm{~mm}$ diameter) was used, and placed using a front loading technique. The balloon-stent combination was unstable during antegrade passage through a transvenous $12 \mathrm{~F}$ sheath. It was therefore positioned in the distal end of the sheath outside of the patient, and the entire sheath, balloon, and stent combination was then passed transvenously as a unit to the stenosis, where the stent was successfully deployed.

Four patients had significant narrowing of both pathways. In three patients, both stents were placed uneventfully at a single procedure (fig 1), but in one patient the stenoses were very close together and were dealt with separately six weeks apart.

In two patients the P308 stent displaced distally along the balloon during inflation, resulting in imperfect and unstable stent deployment. The balloon extends considerably further along the catheter than indicated by the radio-opaque markers and in one (early) case, the sheath was incompletely withdrawn and the proximal end of the balloon remained trapped in the sheath, causing uneven balloon inflation and stent malpositioning. The other stent displacement was probably due to excessive shortening of the stent on an oversized balloon $(25 \mathrm{~mm})$. In both cases, it was possible to deflate the balloon, which was then advanced into the stent and partially reinflated to catch it. The balloon-stent combination was then withdrawn over the guidewire to the iliac vein, where the stent was safely fully deployed. The baffle stenosis was successfully stented via the iliac vein stent without further incident. In one further patient, in whom a $23 \mathrm{~mm}$ balloon was used for a P308 stent, there was also excessive shortening of the stent, with eversion of its distal end, but a useful and stable dilatation was achieved and the position was accepted.

In the majority of patients $(\mathrm{n}=16) 11 \mathrm{~F}$ diameter sheaths (William Cook) were used for ease of stent passage, but $9 \mathrm{~F}$ sheaths were used successfully in two patients; one from the internal jugular vein, and one in a smaller child. $12 \mathrm{~F}$ sheaths were used in two patients.

Arrhythmias were relatively common in this group of patients. One young man with a tight inferior pathway stenosis presented after resuscitation from ventricular fibrillation. Electrophysiological study revealed minor sinus node disease only and he has remained asymptomatic since stent insertion (30 months) on amiodarone, digoxin, and enalapril. Two further patients had electrophysiological studies performed during the same procedure as stent insertion. One underwent radiofrequency ablation of atrial flutter and has subsequently had a dual chamber pacing system inserted for sinus node disease. Another patient has also required insertion of a dual chamber pacemaker for sinus node disease. Two patients have continuing complete heart block with satisfactory junctional escape rates at present.

Average fluoroscopy time was 27.4 minutes (11.5-52.5), being longest in the patients with bad stent positioning and retrieval, more than one stent placed, or where there were difficulties with vascular access.

\section{Discussion}

There are substantial numbers of adolescents and young adults with simple TGA who underwent a Mustard-type atrial redirection procedure in the pre-arterial switch era. Many of these patients are very well and asymptomatic but some do report limitations of effort tolerance. In addition it is likely that all these patients are at risk from both late right ventricular decompensation and sudden, probably arrhythmic, death. Systemic venous pathway narrowing is a known risk factor for sudden death ${ }^{8}$ and may be associated with reduced effort tolerance; it would seem reasonable to strive to maintain optimal long term baffle patency in these patients.

The incidence of SVC pathway narrowing in our patients appears to be much lower than in other series, ${ }^{3517}$ with a higher incidence of inferior stenoses, presumably reflecting differences in operative technique.

Of the twenty patients stented in this series, 10 had previously undergone balloon angioplasty but had angiographically significant restenosis on restudy. In four additional patients with previously noted but untreated angiographic narrowings, these had either progressed or failed to grow in proportion with the patient by the time of repeat study. Comparability with our previously published balloon angioplasty experience ${ }^{12}$ is limited by patient growth but suggests that stenting produces at least comparable immediate improvement in angiographic diameters, probably with some enhancement of results for the more acutely angled inferior pathway narrowing. In addition, stenting produced a notably higher rate of complete abolition of pressure gradients $(10 / 24$ $(42 \%) v 6 / 35$ (17\%), $\mathrm{p}=0.04, \chi^{2}$ test).

For both pathways it seems likely that the greatest gain will be in the long term patency of stented baffle narrowings, although longer term follow up will be required to prove this.

\section{TECHNICAL CONSIDERATIONS}

Effective dilatation of systemic venous pathway narrowing was possible using stents in all those in whom it was attempted, and predilatation to test dilatabililty appears unnecessary. Overdilatation of the P308 stents occurs with balloon diameters $>20 \mathrm{~mm}$ and is associated with bad deployment; larger diameter and longer stents are available and should be used if pathway diameters $>20 \mathrm{~mm}$ are required. Front loading of the larger stents may reduce the likelihood of stent migration along the balloon during passage to the site of stenosis, and might also permit the use of smaller diameter sheaths.

Stenting of both superior and inferior caval pathways during the same procedure is technically feasible, but where stenoses are closely approximated it may occasionally be prudent to treat the sites at separate procedures.

Badly deployed stents can, in general, be safely retrieved from the heart using a partially inflated balloon. It is probably preferable to redeploy such stents in the iliac vein, rather than attempting percutaneous extraction, although the long term effects of this are unknown. 
Given the metal content of implanted stents, it would seem sensible to perform any electrophysiological studies or radiofrequency ablation before stent insertion. However, the converse is probably true for insertion of transvenous pacing systems. There are reports of both SVC syndrome and sudden death from SVC obstruction following pacemaker insertion, ${ }^{8}{ }^{11}$ which might be avoided with more permanently improved caval dimensions and passage of the pacing wire(s) via the stent lumen.

CLINICAL CONSIDERATIONS

In this retrospective, observational study, it was difficult to address the issues of either clinical outcome or of right ventricular performance. Although the majority of patients described a subjective improvement in effort tolerance following stenting we have no objective evidence for this, and only five patients had complaints before the procedure (two mainly arrhythmic). None of our patients had overt SVC syndromes from which more significant symptomatic benefit might be expected. In addition, five patients concurrently started ACE inhibitor treatment. Relief of baffle stenosis is unlikely to have any beneficial effect on right ventricular function and it is noteworthy that four patients have continued their progressive and severe deterioration in both right ventricular function and clinical status. It seems more likely that any improvement in longevity will result from reduced susceptibility to sudden arrhythmic death than from improved ventricular performance, and certainly the patient presenting in ventricular fibrillation has had no further symptomatic arrhythmias.

A number of groups have advocated pulmonary artery banding, followed by takedown of the Mustard operation and arterial switching as an alternative to transplantation for the failing right ventricle. ${ }^{18}{ }^{19}$ Clearly, intracardiac stents may complicate takedown of the Mustard baffles at the time of late switching, but nevertheless may be required to allow the patient to survive the preparatory pulmonary artery banding.

CONCLUSIONS

Stenting of systemic venous pathway obstruction following Mustard repair of TGA is safe and is probably more effective than balloon angioplasty alone, particularly in offering improved prospects for the maintenance of unobstructed systemic venous pathways in the longer term. Improved long term baffle patency should reduce the risks from tachyarrhythmias and may improve effort tolerance, as well as improving access for pacing. However, both the absolute indications for pathway stenting and the effects on long term outcome require further evaluation.

1 Mustard WT. Successful two-stage correction of transposition of the great vessels. Surgery 1964;55:469-72.

2 Aghaji MAC, Litwin SB. Results of Mustard's repair for dextro-transpostic

3 Marx GR, Hougen TJ, Norwood WI. Transposition of the great arteries with intact ventricular septum: results of great arteries with intact ventricular septum: results of patients. F Am Coll Cardiol 1983;1:476-83.

4 Myridakis DJ, Ehlers KH, Engle MA. Late follow-up after venous switch operation (Mustard procedure) for simple and complex transposition of the great arteries. Am f Cardiol 1994;74:1030-6.

5 Stark J, Silove ED, Taylor JFN, et al. Obstruction to systemic venous return following Mustard's operation for transposition of the great arteries. F Thorac Cardiovasc Surg 1974;68: $742-9$.

6 Bowyer JJ, Busst CM, Till JA, et al. Exercise ability after Mustard's operation. Arch Dis Child 1990;65:865-70.

7 Kirk CR, Gibbs JL, Wilkinson JL, et al. Protein losing enterKirk CR, Gibbs JL, Wilkinson JL, et al. Protein losing enter-
opathy caused by baffle obstruction after Mustard's operation. Br Heart f 1988;59:69-72.

8 Gewillig M, Cullen S, Mertens B. Risk factors for arrhythmia and death after Mustard's operation for simple transposition of the great arteries. Circulation 1991; 84(supp III): 187-92.

9 Deanfield J, Camm J, Macartney F, et al. Arrhythmia and late mortality after Mustard and Senning operations for ransposition of the great arteries. F Thorac Cardiovasc Surg 1988;96:569-76.

10 Park, SC, Neches WH, Mathews RA. Haemodynamic function after the Mustard operation for transposition of the great arteries. Am f Cardiol 1973;51:1514-19.

11 Lock JE, Bass JL, Castenada-Zuni GAW, et al. Dilation angioplasty of congenital or operative narrowings of venous channels. Circulation 1984;70:457-64.

12 Sreeram N, Dikkala V, Arnold R. Balloon dilation of stenosed systemic venous pathways following the Mustard operation. Cardiology in the Young 1995;5:238-42.

13 Chatelain P, Meier B, Friedli B. Stenting of superior and inferior vena cava for symptomatic narrowing after repeated surgery for D-transposition of the great arteries. Br Heart F 1991;66:466-8.

14 Ward CJB, Mullins CE, Nihill MR, et al. Use of intravascular stents in systemic venous and systemic venous baffle obstructions. Circulation 1995;91:2948-54.

15 Shuping G, Shiota T, Rice MJ, et al. Transoesophageal ultrasound imaging during stent implantation to relieve superior vena cava to intra-atrial baffle obstruction after Mustard repair of transposition of the great arteries. Circulation 1995;91:2679-80.

16 Kaulitz R, Stumper OFW, Geuskens R, et al. Comparative values of the precordial and transesophageal approaches in the echocardiographic evaluation of atrial baffle function after an atrial correction procedure. $\mathcal{F} \mathrm{Am}$ Coll Cardiol 1990;16:686-94.

17 Williams WG, Trusler GA, Kirklin JW, et al. Early and late results of a protocol for simple transposition leading to and atrial switch (Mustard) repair. F Thorac Cardiovasc Surg 1988;95:717-26.

18 Chang AC, Wernovsky G, Wessel DL et al. Surgical management of late right ventricular failure after Mustard or Senning repair. Circulation 1992;86(suppl 5):140-9.

19 Cochrane AD, Karl TR, Mee RB. Staged conversion to arterial switch for late failure of the systemic right ventricle. Ann Thorac Surg 1993;56:854-61. 